\title{
Cationic Contrast Agent Diffusion Differs Between Cartilage and Meniscus
}

\author{
Juuso T. J. Honkanen, ${ }^{1,2}$ Mikael J. Turunen, ${ }^{1}$ Jonathan D. Freedman, ${ }^{3}$ Simo Saarakkala, $, 4,6$ \\ Mark W. Grinstaff, ${ }^{7,8}$ Janne H. Ylärinne, ${ }^{9}$ Jukka S. Jurvelin, ${ }^{1,2}$ and Juha Töyräs ${ }^{1,2}$ \\ ${ }^{1}$ Department of Applied Physics, University of Eastern Finland, POB 1627, 70211 Kuopio, Finland; ${ }^{2}$ Diagnostic Imaging \\ Center, Kuopio University Hospital, Kuopio, Finland; ${ }^{3}$ Department of Pharmacology, Boston University School of Medicine, \\ Boston, MA, USA; ${ }^{4}$ Research Unit of Medical Imaging, Physics and Technology, University of Oulu, Oulu, Finland; \\ ${ }^{5}$ Department of Diagnostic Radiology, Oulu University Hospital, Oulu, Finland; ${ }^{6}$ Medical Research Center Oulu, Oulu \\ University Hospital and University of Oulu, Oulu, Finland; ${ }^{7}$ Department of Biomedical Engineering, Boston University, Boston, \\ MA, USA; ${ }^{8}$ Department of Chemistry, Boston University, Boston, MA, USA; and ${ }^{9}$ Department of Integrative Medical Biology, \\ University of Umea, Umeå, Sweden
}

(Received 22 January 2016; accepted 22 April 2016; published online 29 April 2016)

Associate Editor Sean S. Kohles oversaw the review of this article.

\begin{abstract}
Contrast enhanced computed tomography (CECT) is a non-destructive imaging technique used for the assessment of composition and structure of articular cartilage and meniscus. Due to structural and compositional differences between these tissues, diffusion and distribution of contrast agents may differ in cartilage and meniscus. The aim of this study is to determine the diffusion kinematics of a novel iodine based cationic contrast agent $\left(\mathrm{CA}^{2+}\right)$ in cartilage and meniscus. Cylindrical cartilage and meniscus samples $(d=6 \mathrm{~mm}$, $h \approx 2 \mathrm{~mm}$ ) were harvested from healthy bovine knee joints $(n=10)$, immersed in isotonic cationic contrast agent $(20 \mathrm{mgI} / \mathrm{mL})$, and imaged using a micro-CT scanner at 26 time points up to $48 \mathrm{~h}$. Subsequently, normalized X-ray attenuation and contrast agent diffusion flux, as well as water, collagen and proteoglycan (PG) contents in the tissues were determined. The contrast agent distributions within cartilage and meniscus were different. In addition, the normalized attenuation and diffusion flux were higher $(p<0.05)$ in cartilage. Based on these results, diffusion kinematics vary between cartilage and meniscus. These tissue specific variations can affect the interpretation of CECT images and should be considered when cartilage and meniscus are assessed simultaneously.
\end{abstract}

Keywords-Computed tomography, Contrast enhancement, Diffusion flux, Diffusion kinematics, Normalized attenuation.

Address correspondence to Juuso T. J. Honkanen, Department of Applied Physics, University of Eastern Finland, POB 1627, 70211 Kuopio, Finland. Electronic mails: juuso.honkanen@uef.fi, mikael.turunen@uef.fi, jonathanfreedman727@gmail.com, simo. saarakkala@oulu.fi,mgrin@bu.edu,janne.ylarinne@umu.se, jukka. jurvelin@uef.fi, and juha.toyras@uef.fi

\section{INTRODUCTION}

Articular cartilage and meniscus are crucial tissue structures for the healthy functioning of the knee joint. Articular cartilage is an avascular specialized connective tissue with inhomogeneous structure that covers the ends of articulating bones. It enables low-friction joint movement, and together with meniscus, distributes loads in the knee joint. ${ }^{7,10}$ The crescent shaped menisci are mostly avascular fibrocartilaginous tissues located between the medial and lateral condyles of femur and tibial plateaus. In addition to load distribution, menisci stabilize the joint, absorb shocks, and improve joint lubrication. ${ }^{10,12,13}$ Structural constituents of these tissues are similar, but their contents and tissue structures differ significantly. The extracellular matrix of cartilage and meniscus consists of water (68-85\% vs. 60-75\%), proteoglycans (PGs) $(5-10 \%$ vs. $<1-2 \%$ ), and collagens $(10-20 \%$, mainly type II vs. $15-25 \%$, mainly type I), respectively. ${ }^{10,27}$ Due to the avascular nature of these tissues, the transport of nutrients occurs primarily via diffusion and convection from the synovial fluid, thus hindering the regeneration of cartilage and meniscus. ${ }^{10,11,16,26,28}$

Contrast enhanced computed tomography (CECT) is a non-destructive imaging technique to examine the biochemical composition, structure, and integrity of articular cartilage. From a clinical perspective, assessment of both cartilage and meniscus is key for successful diagnosis and treatment of degenerative diseases, e.g. osteoarthritis (OA). In order to address this imaging need, CECT has 
widely been investigated for the detection of cartilage degeneration and lesions, ${ }^{18,20,34,38}$ as well as, recently, to the imaging of bovine and human meniscus. ${ }^{14,24}$

CECT requires the use a contrast agent and commonly employs anionic contrast agents such as ioxaglate $(q=-1)$ or gadopentate $(q=-2),{ }^{25}$ which distribute mainly by diffusion into the tissue and equilibrate inversely to the spatial distribution of the negatively charged PGs. ${ }^{4,35}$ In addition to PG content and distribution, contrast agent diffusion and distribution are influenced by the water content, collagen content, and structural integrity of the tissue matrix. ${ }^{9,19,21,36}$ Recently, novel cationic contrast agents were introduced for CECT imaging of cartilage $e^{2,17,37}$ and meniscus. ${ }^{24}$ As the cationic contrast agent molecules are attracted by the negative fixed charge density (FCD) of the tissues, CECT using cationic contrast agents offers a sensitive technique for direct monitoring of changes in cartilage and meniscus PG contents. Indeed, previous studies describe strong positive correlations between contrast agent distribution of a cationic contrast agent bearing four positive charges $\left(\mathrm{CA}^{4+}\right)$ and $\mathrm{PG}$ content in these tissues. ${ }^{3,23,24}$

CECT has the potential to become a quantitative laboratory or clinical tool for assessment of knee joint composition and condition, given that articular cartilage and meniscus can be assessed simultaneously. Thus, it is important to determine the diffusion kinematics of the cationic contrast agent in cartilage and meniscus. Since the composition and structure of cartilage and meniscus are different, we hypothesize that the diffusion characteristics of the cationic contrast agent will vary between these tissues. Moreover, the previous studies mentioned above used $\mathrm{CA}^{4+}$ with a molecular weight of $1355 \mathrm{~g} / \mathrm{mol}$, and we rationalized that a smaller $\left(686 \mathrm{~g} / \mathrm{mol}, \mathrm{CA}^{2+}\right)$ but still cationic contrast agent may have a greater utility for CECT, as it should exhibit shorter times to equilibrium. Thus, the aim of this ex vivo study was to compare the diffusion of a smaller molecular weight cationic contrast agent bearing two positive charges, $\mathrm{CA}^{2+}$, into articular cartilage and meniscus. Herein, we report the: (1) CECT of bovine cartilage and meniscus plugs using a micro-CT scanner; (2) determination of the time to equilibrium and diffusion flux for $\mathrm{CA}^{2+}$ in both cartilage and meniscus tissues; (3) distribution of the cationic contrast agent within these tissues; and (4) dependence of these values on compositional (PG, water, hydroxyproline, and uronic acid) characteristics.

\section{MATERIALS AND METHODS}

\section{Sample Preparation}

Similarly to our previous study, ${ }^{15}$ skeletally mature bovine knees ( $n=10$, one knee joint per animal) were obtained from a local slaughterhouse (HK Ruokatalo Oy, Outokumpu, Finland) within 24 h of slaughtering. Osteochondral plugs $(d=25.4 \mathrm{~mm})$ were drilled from the upper lateral quadrant of the patellae (Fig. 1a). The plug was cut into quadrants and from one quadrant a sample plug $(d=6.0 \mathrm{~mm})$ was punched out. Additionally, approximately $2.0 \mathrm{~mm}$ thick plug $(d=6.0 \mathrm{~mm})$ from the central region of the medial meniscus was detached (Fig. 1b). One osteochondral and one meniscal plug were harvested from each joint. Surrounding tissue of the cartilage and meniscus plugs was collected for compositional analysis (Figs. 1a and $1 b)$. Only healthy tissues, i.e., no visible signs of surface fibrillation or other early degenerative changes, were harvested.

\section{CECT Imaging}

The edges of the osteochondral and meniscus plugs were carefully sealed with a thin film of cyanoacrylate and glued on the bottom of plastic tubes. The tubes were filled with phosphate buffered saline (PBS) to completely immerse the plugs and a non-contrast image was acquired at room temperature. Subsequently, the PBS was carefully removed and replaced

(a)

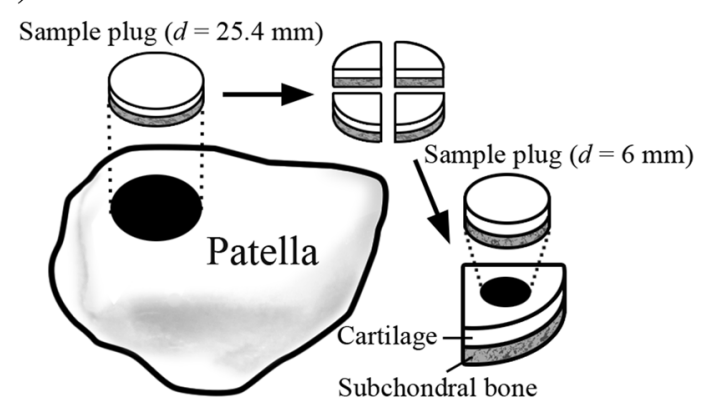

(b)

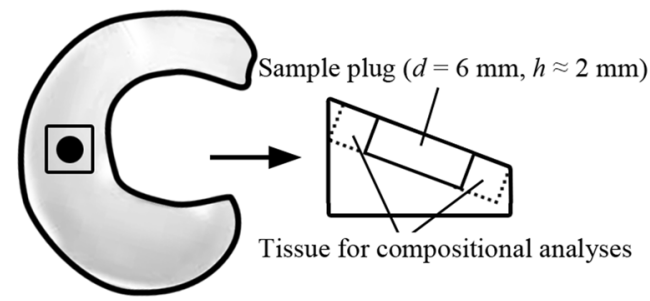

FIGURE 1. (a) An osteochondral plug $(d=25.4 \mathrm{~mm})$ was drilled from the upper lateral quadrant of a healthy bovine patella and trimmed to have $\sim 2 \mathrm{~mm}$ bone under the cartilage. Subsequently, the disk was cut into four similar sections and a smaller osteochondral plug $(d=6 \mathrm{~mm})$ was punched from a section with the flattest cartilage surface. The tissue surrounding the $6 \mathrm{~mm}$ plug was harvested for compositional analyzes. (b) A cylindrical plug $(d=6 \mathrm{~mm})$ from the central region of medial bovine meniscus was harvested and trimmed to be $2 \mathrm{~mm}$ in height. In addition, the surrounding tissue (rectangular area $\sim 2 \mathrm{~mm}$ around the plug, $h \approx 2 \mathrm{~mm}$ ) was harvested for compositional analyzes. 
with a novel iodine based cationic contrast agent $\left(\mathrm{CA}^{2+}\right)(q=+2, M=686 \mathrm{~g} / \mathrm{mol})^{17}$ diluted with PBS including penicillin-streptomycin $\left(100\right.$ units $\mathrm{mL}^{-1}$ penicillin, $100 \mu \mathrm{g} \mathrm{mL}^{-1}$ streptomycin; EuroClone, Siziano, Italy), antimycotic agent (Gibco Fungizone Antimycotic, $250 \mu \mathrm{g} \mathrm{mL}^{-1}$ amphotericin B, $205 \mu \mathrm{g}$ $\mathrm{mL}^{-1}$ sodium deoxycholate; Life Technologies, Carlsbad, CA, USA) and proteolytic inhibitors [5 mM ethylenediaminetetraacetic acid disodium salt (EDTA; VWR International, Fontenay, France) and $5 \mathrm{mM}$ benzamidine hydrochloride hydrate (Sigma-Aldrich Inc., St. Louis, MO, USA)] to $20 \mathrm{mgI} / \mathrm{mL}$ concentration. Both plugs were imaged for $48 \mathrm{~h}$ in isotonic contrast agent bath $(330 \mathrm{mOsm} / \mathrm{kg}, 2 \mathrm{~mL})$ at the following time points: $10,20,30,40,50,60,70,8090$ and $100 \mathrm{~min}$, and 2, 2.5, 3, 3.5, 4, 4.5, 5, 6, 7, 16, 20, 24, 28, 44, 46 and $48 \mathrm{~h}$. CECT imaging was performed using a micro-CT-scanner (SkyScan 1172, SkyScan, Kontich, Belgium) with an isotropic voxel size of $25 \mu \mathrm{m} \times$ $25 \mu \mathrm{m} \times 25 \mu \mathrm{m}, 100 \mathrm{kV}$ tube voltage, and acquisition time of $10 \mathrm{~min}$. Since the edges of the plugs were sealed, contrast agent penetration was allowed only through the articulating surface. After $48 \mathrm{~h}$ of contrast agent immersion the $\mathrm{CA}^{2+}$ was washed out by immersing the plugs in PBS for $48 \mathrm{~h}$ at $9^{\circ} \mathrm{C}$. Subsequently, the plugs were fixed in $10 \%$ formalin, dehydrated in ascending series of ethanol, decalcified (cartilage + bone) and embedded in paraffin to enable cutting the sections for histological analysis.

Micro-CT data-analysis was conducted using custom made MATLAB scripts (R2012a, MathWorks, Inc., Natick, MA, USA). The image stacks for each time point were co-registered. 51 subsequent coronal slices were averaged to enhance the signal-to-noise- ratio. A similar procedure was done for the non-contrast image, which was subtracted from the CECT images. A $1275 \mu \mathrm{m}$ wide region (51 pixels) of interest (ROI), height matched with full thickness of the tissue, was selected in the center of the sample. The pixel rows in the ROI were horizontally averaged and the X-ray attenuation was normalized with that in the surrounding contrast agent bath to obtain a depth-wise normalized attenuation (i.e. contrast agent partition) profile (Fig. 2).

Time dependent normalized attenuation was determined by fitting an exponential function to the bulk X-ray attenuation values, $C(\%)$, determined at different time points $t(\mathrm{~s})$ :

$$
C=a e^{-b t}+c,
$$

where $a, b$, and $c$ are the fitting coefficients. ${ }^{24}$ The time required to reach equilibrium was determined as the time at which the change in the normalized attenuation was less than $0.05 \%$ per hour. ${ }^{15}$ The diffusion flux, $J$ $\left(\mathrm{mol} / \mathrm{m}^{2} / \mathrm{s}\right)$, through the tissue surface was calculated as follows:

$$
J=-h \frac{\partial \bar{C}}{\partial t}
$$

where $h(\mathrm{~m})$ is the sample thickness, $t(\mathrm{~s})$ is time and $\bar{C}$ is the bulk contrast agent concentration $\left(\mathrm{mol} / \mathrm{m}^{3}\right)$ within the sample derived from Eq. (1).

\section{Histology and Compositional Analyzes}

The paraffin embedded samples were halved and cut into 3 and $5 \mu \mathrm{m}$ thick sections for analysis of PG and collagen distributions, respectively. Subsequently, the (a)

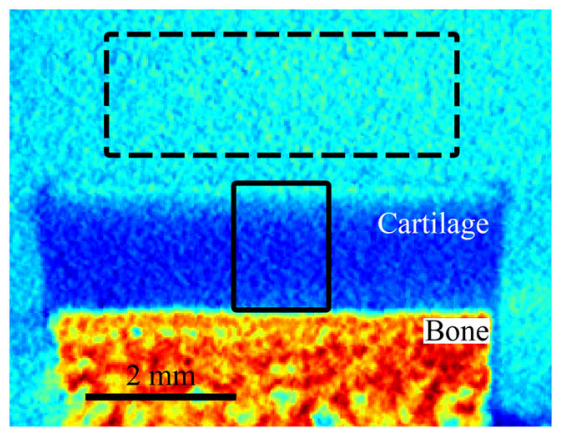

(b)

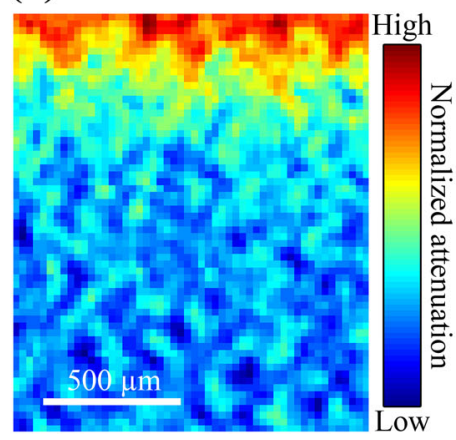

(c)

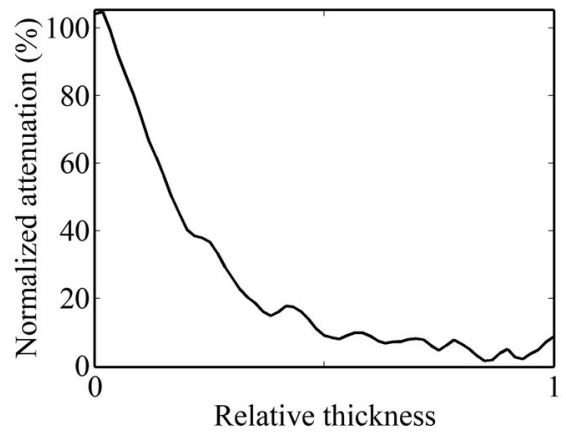

FIGURE 2. (a) A micro-CT image (average of 51 consecutive $25 \mu \mathrm{m}$ thick slices) of one representative cartilage sample after $10 \mathrm{~min}$ of contrast agent diffusion and before background (non-contrast image) subtraction. The analyzed region of interest (ROI) is delineated with a solid line and the ROI for normalization delineated with a dashed line. The width of the analyzed ROI was $1275 \mu \mathrm{m}$ (51 pixels) and the height was matched with the full thickness of the cartilage. An identical approach was used when CECT images of the menisci were analyzed. Scale bar $=2 \mathrm{~mm}$. (b) A magnified image of the analyzed cartilage area after background subtraction. Scale bar $=500 \mu \mathrm{m}$. (c) A depth-wise contrast agent distribution profile. The horizontal axis represents the relative distance from the surface (0) to the cartilage-bone interface (1). To create the distribution profiles the pixel rows in subfigure (b) were horizontally averaged and normalized with the average X-ray attenuation value within the normalization ROI shown in the subfigure (a). 
paraffin was dissolved and the $3 \mu \mathrm{m}$ sections were stained with Safranin-O. The spatial distribution of the stain in the section was measured using quantitative digital densitometry (DD) revealing the PG distribution. ${ }^{31}$ Three sections per sample $(1275 \mu \mathrm{m}$ in width, full thickness) were analyzed and the depth-wise profiles were averaged. The measurements were done utilizing a light microscope (Nikon Microphot-FXA, Nikon Co., Tokyo, Japan), equipped with a monochromatic light source and a 12-bit CCD camera (ORCA-ER, Hamamatsu Photonics K.K., Hamamatsu, Japan). The system was calibrated with neutral density filters (Schott, Mainz, Germany) covering optical density (OD) range from 0 to 2.6 .

The collagen distribution in $5 \mu \mathrm{m}$ sections, adjacent to the ones used in DD, was determined using Fourier transform infrared (FTIR) microspectroscopy (Hyperion 3000, Bruker Corporation, Billerica, MA, USA). After deparaffinization, the sections were moved on Zinc-Selenide ( $\mathrm{ZnSe}$ ) IR-windows. A $1 \mathrm{~mm}$ wide, full sample thickness ROI was determined from three sections per sample. Data was collected within wavenumber range of $2000-720 \mathrm{~cm}^{-1}$ using a pixel size of $20 \times$ $20 \mu \mathrm{m}^{2}$ and spectral resolution of $8 \mathrm{~cm}^{-1}$. The background was measured on a clean $\mathrm{ZnSe}$-window using the same parameters as above. Collagen content in each pixel was determined as the amide I (1720$1595 \mathrm{~cm}^{-1}$ ) peak area. ${ }^{32}$ The depth-wise profiles of collagen content were calculated for each section and the three profiles were averaged.

Water, hydroxyproline and uronic acid contents (the latter two corresponding to collagen and PG contents, respectively) were determined from the tissues surrounding the sample plugs using compositional analyzes. Water content was determined via lyophilization. Hydroxyproline and uronic acid contents were determined from the plugs digested with $1 \mathrm{mg} / \mathrm{mL}$ concentration of papain in $150 \mathrm{mM}$ sodium acetate including $50 \mathrm{mM}$ Cys- $\mathrm{HCl}$ and $5 \mathrm{mM}$ EDTA at $\mathrm{pH}$ of 6.5 in $60^{\circ} \mathrm{C}$ for $3 \mathrm{~h}$ to digest the PGs. Enzyme inactivation was managed by boiling the sections for $10 \mathrm{~min}$. Subsequently, the hydroxyproline content was deter- mined from the freeze dried and papain digested sections with spectrophotometric assay. ${ }^{6}$ The uronic acid content was quantified from the ethanol-precipitated samples dissolved in water. ${ }^{5}$ The contents were determined three times for each sample, normalized by the sample wet weights and averaged.

\section{Statistical Analyzes}

Wilcoxon signed rank test was used to determine the significance of the differences between the parameter values of cartilage and meniscus. The Wilcoxon signed rank test was chosen due to the relatively low number of paired samples. Spearman's rho was determined to analyze the significance of relationships between the normalized attenuation and reference parameters (i.e. water, uronic acid and hydroxyproline contents and bulk OD values). Multiple linear regression analysis was conducted between the compositional parameters (i.e., water, hydroxyproline and uronic acid contents) and the normalized attenuation of pooled samples. The statistical tests were conducted using SPSS (v. 21.0.0.0, SPSS Inc., IBM Company, Armonk, NY, USA).

\section{RESULTS}

After $48 \mathrm{~h}$ of diffusion, the normalized attenuation reached $289.4 \pm 44.2 \%$ in cartilage and $159.7 \pm$ $11.2 \%$ in the meniscus (Table 1; Fig. 3a). At all time points after $50 \mathrm{~min}$, the normalized attenuation was significantly higher $(p<0.05)$ in cartilage than in meniscus (Figs. 3a and 4; Table 1). The diffusion flux was significantly higher $(p<0.05)$ in cartilage at all time points (Fig. 3b). However, no significant differences $(p=1.0)$ were found in the time to reach equilibrium for $\mathrm{CA}^{2+}$ in cartilage and meniscus $(35.8 \pm 6.5$ and $37.3 \pm 9.6 \mathrm{~h}$, respectively). The contrast agent distribution within the cartilage and meniscus sample plugs was different (Figs. 4, 5a and $5 b)$. Moreover, the majority of $\mathrm{CA}^{2+}$ accumulated in the deep zone of cartilage, while in the meniscus, the

TABLE 1. Normalized attenuation at 1 and $48 \mathrm{~h}$ time points, water, hydroxyproline and uronic acid contents and optical densities of bovine cartilage $(n=10)$ and meniscus $(n=10)$.

\begin{tabular}{|c|c|c|c|c|c|c|c|}
\hline & $\begin{array}{l}\text { Normalized } \\
\text { attenuation } \\
(\%), 1 \mathrm{~h}\end{array}$ & $\begin{array}{c}\text { Normalized } \\
\text { attenuation } \\
(\%), 48 \mathrm{~h}\end{array}$ & Water (\%) & $\begin{array}{l}\text { Hydroxyproline } \\
(\mu \mathrm{g} / \mathrm{mg}, \mathrm{w} . \mathrm{w} .)\end{array}$ & $\begin{array}{l}\text { Uronic acid } \\
(\mu \mathrm{g} / \mathrm{mg}, \mathrm{w} . \mathrm{w} .)\end{array}$ & $\begin{array}{l}\text { Optical } \\
\text { density }\end{array}$ & $\begin{array}{l}\text { Thickness } \\
(\mathrm{mm})\end{array}$ \\
\hline Cartilage & $\begin{array}{l}57.5 \pm 16.6^{*} \\
(22.8,80.1)\end{array}$ & $\begin{array}{l}289.4 \pm 44.2^{*} \\
(225.0,340.3)\end{array}$ & $\begin{array}{l}80.4 \pm 2.4^{*} \\
(76.6,83.1)\end{array}$ & $\begin{array}{l}15.4 \pm 2.3^{*} \\
(11.0,19.0)\end{array}$ & $\begin{array}{l}9.0 \pm 3.4^{*} \\
(4.4,14.8)\end{array}$ & $\begin{array}{l}1.66 \pm 0.09^{*} \\
(1.47,1.75)\end{array}$ & $\begin{array}{l}2.01 \pm 0.33 \\
(1.55,2.58)\end{array}$ \\
\hline Meniscus & $\begin{array}{l}39.5 \pm 12.0^{*} \\
(21.4,60.2)\end{array}$ & $\begin{array}{l}159.7 \pm 11.2^{*} \\
(141.0,178.2)\end{array}$ & $\begin{array}{l}72.1 \pm 1.8^{*} \\
(69.3,75.1)\end{array}$ & $\begin{array}{l}33.7 \pm 3.4^{\star} \\
(26.7,39.8)\end{array}$ & $\begin{array}{l}3.3 \pm 0.5^{\star} \\
(2.4,3.9)\end{array}$ & $\begin{array}{l}0.88 \pm 0.24^{*} \\
(0.52,1.16)\end{array}$ & $\begin{array}{l}1.83 \pm 0.30 \\
(1.45,2.25)\end{array}$ \\
\hline
\end{tabular}

Mean values $( \pm S D)$ of the parameters are presented in the top row and the range in parentheses ( $\min$, max) in the bottom row.

* Significant difference $(p<0.05)$ between cartilage and meniscus samples, Wilcoxon signed rank test. 
(a)

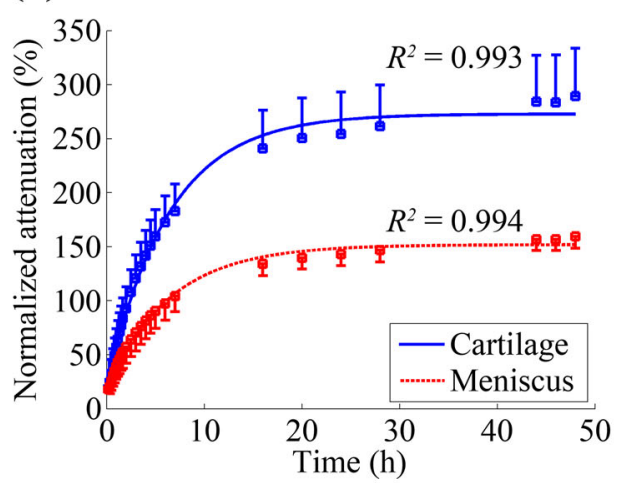

(b)

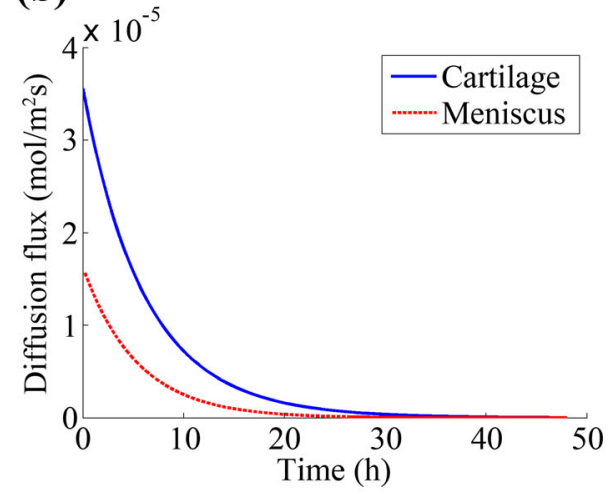

FIGURE 3. (a) Mean ( $n=10)$ normalized attenuation in bovine cartilage (+SD) and meniscus (-SD) at 26 different time points (10, 20, 30, 40, 50, 60, 70, 8090 and $100 \mathrm{~min}$, and 2, 2.5, 3, 3.5, 4, 4.5, 5, 6, 7, 16, 20, 24, 28, 44, 46 and 48 h after immersion). The normalized attenuation was significantly higher $(p<0.05)$ in cartilage than in meniscus at all time points after 50 min. (b) Mean $(n=10)$ diffusion fluxes for cartilage and meniscus as a function of immersion time. The diffusion flux was significantly higher $(p<0.05)$ in cartilage than in meniscus at all time points.

$\mathrm{CA}^{2+}$ accumulated closer to the articulating surface (Figs. 3, 5a and 5b).

Water and uronic acid contents and bulk OD values were significantly higher $(p=0.005$ for all $)$ and hydroxyproline content was significantly lower $(p=0.005)$ in cartilage than in meniscus (Table 1). The depth-wise PG distribution was similar, while the depth-wise collagen content distribution was different between the tissues (Fig. 5). There was no significant $(p>0.05)$ relationship between the normalized attenuation and water, uronic acid, and hydroxyproline contents as well as bulk OD values within cartilage or meniscus sample pools. However, when samples from both tissues were pooled $(n=20)$, the composition significantly predicted the normalized attenuation at diffusion equilibrium $(48 \mathrm{~h}): F(3,16)=27.934, p<0.001$, $R^{2}=0.84$. However, only uronic acid was significant $(p<0.05)$ predictor of the normalized attenuation.

\section{DISCUSSION}

The aim of this study was to investigate the diffusion kinematics of cationic contrast agent $\left(\mathrm{CA}^{2+}\right)$ in healthy bovine articular cartilage and meniscus. Accordingly, we determined the normalized attenuation at 26 different time points over $48 \mathrm{~h}$ of contrast agent diffusion. Subsequently, the diffusion flux was determined. Normalized attenuation was found to be significantly higher in cartilage than in meniscus after 50 min of diffusion. Furthermore, the diffusion flux was systematically higher in cartilage throughout the whole experiment. However, no statistically significant difference was observed between the tissues in the time required to reach the diffusion equilibrium.
The normalized attenuation at equilibrium was $>100 \%$ in both tissues compared with the initial bath attenuation. This is due to accumulation of cationic contrast agent molecules in the tissue, due to the electrostatic attraction between PGs and contrast agent molecules. With anionic contrast agents, instead, the normalized attenuation (i.e. contrast agent partition) at diffusion equilibrium is $<100 \%,{ }^{14,15,18,22,30}$ as the anionic contrast agents are repelled by the negative FCD of the tissues. The normalized attenuation was significantly higher in cartilage than in meniscus, which reflects the higher PG and water contents in cartilage. These results are consistent with previous studies using the $\mathrm{CA}^{4+}$ cationic contrast agent which reported a strong positive relationship between the normalized attenuation and PG content in cartilage and meniscus. ${ }^{3,23,24}$ In addition, solute diffusivity has been shown to correlate positively with water content in cartilage. ${ }^{9}$

At diffusion equilibrium, most of the contrast agent accumulated in the deep cartilage $(50-94 \%$ of the thickness $\left.{ }^{1,8,16,39}\right)$, i.e., in zone with the highest PG concentration. In the meniscus, the highest contrast agent accumulation was in the lamellar layer (150$200 \mu \mathrm{m}$ thick layer $\sim 10 \mu \mathrm{m}$ beneath the surface ${ }^{33}$ ). Interestingly, the distribution of cationic contrast agent within the meniscus was similar to that reported previously for an anionic contrast agent. ${ }^{15}$ Possibly, this finding could be related to higher water content in the surface and lamellar layers of the meniscus, making these regions more accessible for the $\mathrm{CA}^{2+}$ molecules. The high water content in these layers is also suggested by lower PG and collagen concentrations compared with those in the central layer of the meniscus. Furthermore, the steric hindrance may be greater in the meniscus than in cartilage as the hydroxyproline con- 

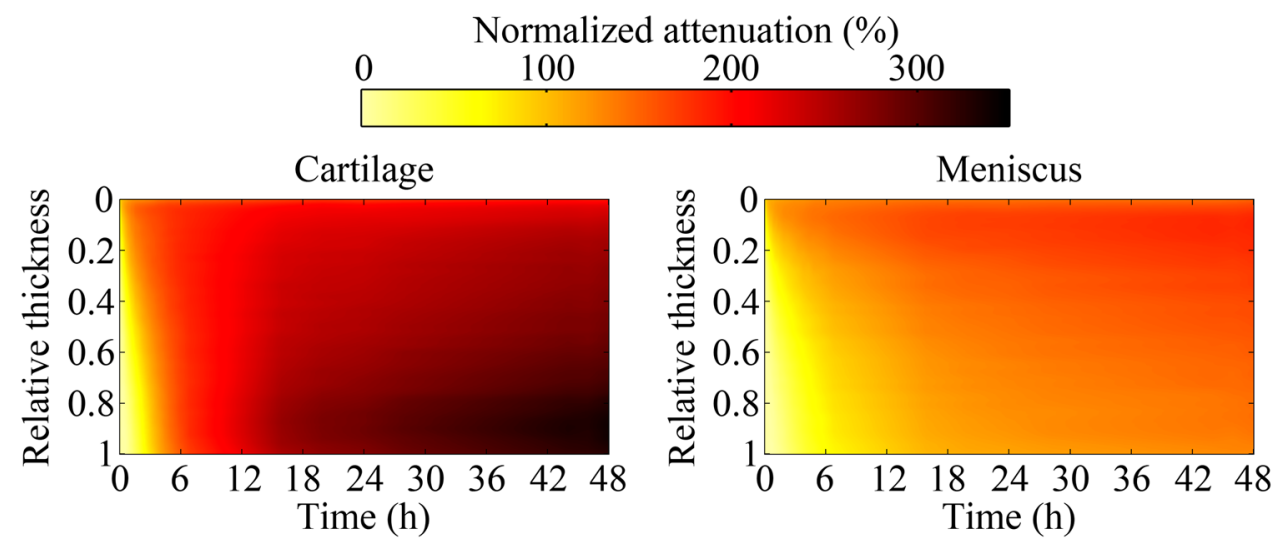

FIGURE 4. Mean $(n=10)$ depth-wise contrast agent distribution within cartilage (left) and meniscus (right) through the experiment. Values at vertical axis represent the relative thickness of the samples, 0 corresponds to the articular surface and 1 to the bottom of the tissue.

(a)

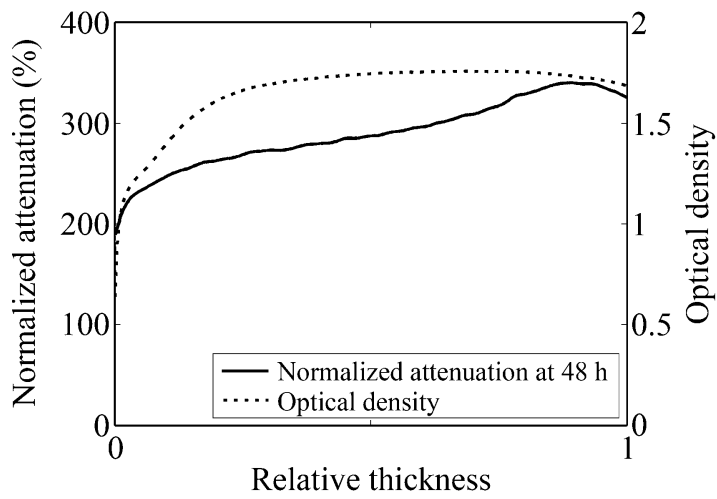

(c)

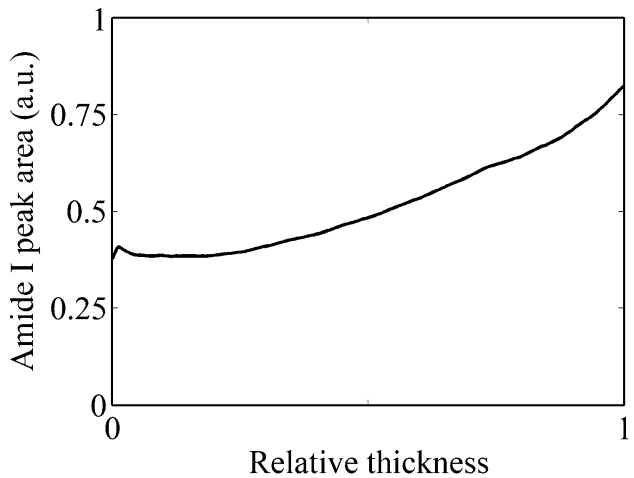

(b)

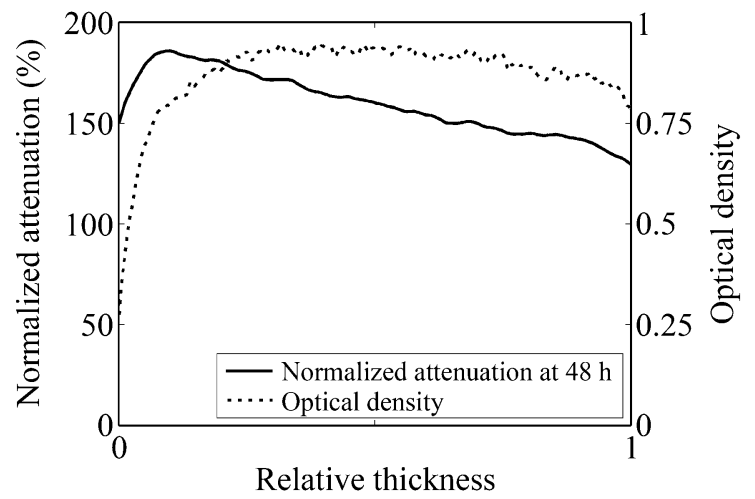

(d)

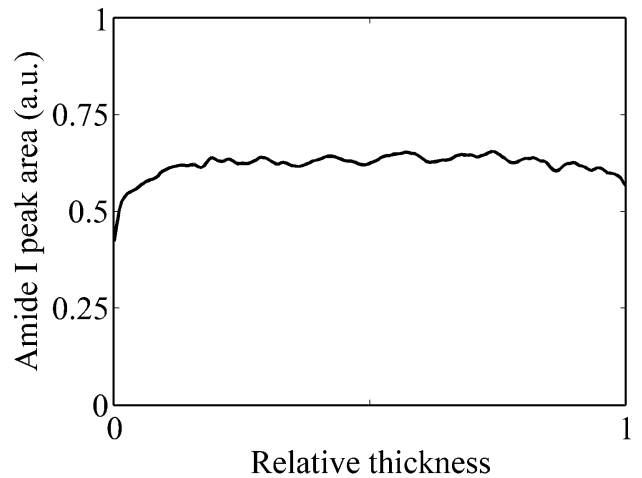

FIGURE 5. Mean $(n=10)$ depth-wise optical density profile (dashed line) corresponding to PG distribution and contrast agent distribution (solid line) in cartilage (a) and meniscus (b) after $48 \mathrm{~h}$ of immersion in cationic contrast agent. Mean $(n=10)$ depthwise amide I peak area corresponding to the collagen distribution in cartilage (c) and meniscus (d). The horizontal axis represents the relative distance from the articular surface (0) to the bottom (1) of the tissue.

tent of the meniscus was over $200 \%$ of that in cartilage. This could hinder accumulation of $\mathrm{CA}^{2+}$ in the central layer of the meniscus.

The diffusion flux was significantly higher in cartilage than in meniscus, due to the differences in the PG and collagen concentrations and distributions in the tissues. The higher PG concentration of cartilage, as compared with that of the meniscus, creates higher electrostatic attraction to the cationic contrast agent molecules. The diffusion towards the deep cartilage maintains the higher diffusion flux of cartilage also at later time points. Furthermore, due to the higher collagen content, the contrast agent molecules may be more affected by the steric hindrance of the matrix in 
the meniscus than in cartilage. The amount of contrast agent diffused into cartilage was also substantially greater. The majority of $\mathrm{CA}^{2+}$ accumulated close to the articulating surface (i.e., in the lamellar layer) in the meniscus samples. In cartilage samples, $\mathrm{CA}^{2+}$ accumulated in the deep zone, which prolonged the diffusion process. For these reasons, the time required to reach diffusion equilibrium was not different between the tissues, although the diffusion flux was higher in cartilage.

In this study, cylindrical osteochondral and meniscal plugs excised from healthy bovine knee joints were used to study the diffusion kinematics of a cationic contrast agent. Due to the relatively small homogenous sample population ( $n=10$ for cartilage and meniscus), the variation of tissue properties was limited, and no statistical significances were observed between the normalized attenuation and reference parameters in cartilage or meniscus sample pools. However, the homogeneity in sample population allowed a reliable comparison of the diffusion kinematics between cartilage and meniscus tissues.

The main constituents (water, PGs and collagens) are the same in cartilage and meniscus, but their distributions within the tissue differ. However, in case of intact tissues, the amount of contrast agent content at diffusion equilibrium, indicated by the bulk value of normalized attenuation, should be related to the amount of these constituents rather than to their distributions. Thus, the pooling of cartilage and meniscus samples was justified to assess the relationship between contrast agent intake and compositional constituents of the tissues.

As the samples were imaged while immersed in contrast agent bath, diffusion still occurred during the scan. Hence, the normalized attenuation at each time point represents the average value over the scan time rather than precise value at the time point. However, we believe that this had no significant effect on the presented results or conclusions, as the diffusion occurs over many hours.

CECT, using cationic contrast agents, is a promising imaging technique for laboratory purposes, e.g. comprehensive CT-based 3D histopathological evaluation $^{29}$ of the knee joint. However, if an intact knee joint is imaged, the differences in contrast agent diffusion in cartilage and meniscus must be acknowledged when interpreting the results. In addition, the concentration of the contrast agent bath must be maintained at a high level, for example, by intermittent injection of contrast agent. This is because the contrast agent starts to dilute and efflux from the joint capsule. The effluxion takes place in both intact ex vivo and in vivo situations, but is more rapid in vivo due to perfusion. In addition, due to longer diffusion dis- tance, the time required to reach diffusion equilibrium is likely to be longer when full thickness menisci are imaged. The time to reach diffusion equilibrium is too long for clinical applications, even with a cationic contrast agent of small molecular size $(M=686$ $\mathrm{g} / \mathrm{mol})$.

In summary, the distribution of the cationic contrast agent, $\mathrm{CA}^{2+}$, within tissue is different between cartilage and meniscus. The intake of contrast agent is significantly higher in cartilage than in meniscus at all time points after $50 \mathrm{~min}$ of immersion. In addition, the diffusion flux is significantly higher in cartilage at all time points up to $48 \mathrm{~h}$. Nevertheless, the diffusion equilibrium is reached at the same time in both tissues. When both cartilage and meniscus samples are included in the analysis, the compositional parameters predicted the normalized attenuation at $48 \mathrm{~h}$. However, the differences in diffusion kinematics between cartilage and meniscus must be acknowledged in the interpretation of CECT images of knee joint.

CONFLICTS OF INTEREST The authors have no conflicts of interest.

\section{ACKNOWLEDGEMENTS}

Eija Rahunen is acknowledged for histological sample preparation. Krista Rahunen is acknowledged for FTIR imaging. Jan Behm is acknowledged for his assistance in the CECT measurements. Funding from the Academy of Finland (projects 269315 and 268378, JSJ and SS), the strategic funding of University of Eastern-Finland, Kuopio University Hospital (VTR projects 5041746 and 5063535, PY 210, JSJ), European Research Council under the European Union's Seventh Framework Program ((FP/2007-2013)/ERC Grant Agreement no. 336267, SS) and the National Institutes of Health (R01GM098361, MWG) is acknowledged.

\section{REFERENCES}

\footnotetext{
${ }^{1}$ Arokoski, J. P., M. M. Hyttinen, T. Lapveteläinen, P. Takács, B. Kosztáczky, L. Módis, V. Kovanen, and H. Helminen. Decreased birefringence of the superficial zone collagen network in the canine knee (stifle) articular cartilage after long distance running training, detected by quantitative polarised light microscopy. Ann. Rheum. Dis. 55:253-264, 1996.

${ }^{2}$ Bansal, P. N., N. S. Joshi, V. Entezari, B. C. Malone, R. C. Stewart, B. D. Snyder, and M. W. Grinstaff. Cationic contrast agents improve quantification of glycosaminoglycan (GAG) content by contrast enhanced CT imaging of cartilage. J. Orthop. Res. 29:704-709, 2011.
} 
${ }^{3}$ Bansal, P. N., R. C. Stewart, V. Entezari, B. D. Snyder, and M. W. Grinstaff. Contrast agent electrostatic attraction rather than repulsion to glycosaminoglycans affords a greater contrast uptake ratio and improved quantitative CT imaging in cartilage. Osteoarthritis Cartilage 19:970 976, 2011.

${ }^{4}$ Bashir, A., M. L. Gray, R. D. Boutin, and D. Burstein. Glycosaminoglycan in articular cartilage: in vivo assessment with delayed Gd(DTPA)(2-)-enhanced MR imaging. Radiology 205:551-558, 1997.

${ }^{5}$ Blumenkrantz, N., and G. Asboe-Hansen. New method for quantitative determination of uronic acids. Anal. Biochem. 54:484-489, 1973.

${ }^{6}$ Brown, S., M. Worsfold, and C. Sharp. Microplate assay for the measurement of hydroxyproline in acid-hydrolyzed tissue samples. Biotechniques 30:38-40, 2001.

${ }^{7}$ Buckwalter, J. A., and H. J. Mankin. Articular cartilage. Part I: tissue design and chondrocyte-matrix interactions. J. Bone Jt. Surg. Am. 79:600-611, 1997.

${ }^{8}$ Clark, A. L., L. D. Barclay, J. R. Matyas, and W. Herzog. In situ chondrocyte deformation with physiological compression of the feline patellofemoral joint. J. Biomech. 36:553-568, 2003.

${ }^{9}$ Evans, R. C., and T. M. Quinn. Solute diffusivity correlates with mechanical properties and matrix density of compressed articular cartilage. Arch. Biochem. Biophys. 442:1-10, 2005.

${ }^{10}$ Fithian, D. C., M. A. Kelly, and V. C. Mow. Material properties and structure-function relationships in the menisci. Clin. Orthop. Relat. Res. 252:19-31, 1990.

${ }^{11}$ Gershuni, D. H., A. R. Hargens, and L. A. Danzig. Regional nutrition and cellularity of the meniscus. Implications for tear and repair. Sports Med. 5:322-327, 1988.

${ }^{12}$ Ghadially, F. N., J. M. Lalonde, and J. H. Wedge. Ultrastructure of normal and torn menisci of the human knee joint. J. Anat. 136:773-791, 1983.

${ }^{13}$ Herwig, J., E. Egner, and E. Buddecke. Chemical changes of human knee joint menisci in various stages of degeneration. Ann. Rheum. Dis. 43:635-640, 1984.

${ }^{14}$ Honkanen, J. T. J., E. K. Danso, J.-S. Suomalainen, V. Tiitu, R. K. Korhonen, J. S. Jurvelin, and J. Töyräs. Contrast enhanced imaging of human meniscus using cone beam CT. Osteoarthritis Cartilage 23:1367-1376, 2015.

${ }^{15}$ Honkanen, J. T. J., M. J. Turunen, V. Tiitu, J. S. Jurvelin, and J. Töyräs. Transport of iodine is different in cartilage and meniscus. Ann. Biomed. Eng. 2015. doi:10.1007/ s10439-015-1513-2.

${ }^{16}$ Huber, M., S. Trattnig, and F. Lintner. Anatomy, biochemistry, and physiology of articular cartilage. Invest. Radiol. 35:573-580, 2000.

${ }^{17}$ Joshi, N. S., P. N. Bansal, R. C. Stewart, B. D. Snyder, and M. W. Grinstaff. Effect of contrast agent charge on visualization of articular cartilage using computed tomography: exploiting electrostatic interactions for improved sensitivity. J. Am. Chem. Soc. 131:13234-13235, 2009.

${ }^{18}$ Kokkonen, H. T., J. S. Jurvelin, V. Tiitu, and J. Töyräs. Detection of mechanical injury of articular cartilage using contrast enhanced computed tomography. Osteoarthr. Cartil. 19:295-301, 2011.

${ }^{19}$ Kokkonen, H. T., J. Mäkelä, K. A. M. Kulmala, L. Rieppo, J. S. Jurvelin, V. Tiitu, H. M. Karjalainen, R. K. Korhonen, V. Kovanen, and J. Töyräs. Computed tomography detects changes in contrast agent diffusion after collagen cross-linking typical to natural aging of articular cartilage. Osteoarthr. Cartil. 19:1190-1198, 2011.
${ }^{20}$ Kokkonen, H. T., J.-S. Suomalainen, A. Joukainen, H. Kröger, J. Sirola, J. S. Jurvelin, J. Salo, and J. Töyräs. In vivo diagnostics of human knee cartilage lesions using delayed CBCT arthrography. J. Orthop. Res. 32:403-412, 2014.

${ }^{21}$ Kulmala, K. A. M., H. M. Karjalainen, H. T. Kokkonen, V. Tiitu, V. Kovanen, M. J. Lammi, J. S. Jurvelin, R. K. Korhonen, and J. Töyräs. Diffusion of ionic and non-ionic contrast agents in articular cartilage with increased crosslinking-contribution of steric and electrostatic effects. Med. Eng. Phys. 35:1415-1420, 2013.

${ }^{22}$ Kulmala, K. A. M., R. K. Korhonen, P. Julkunen, J. S. Jurvelin, T. M. Quinn, H. Kröger, and J. Töyräs. Diffusion coefficients of articular cartilage for different CT and MRI contrast agents. Med. Eng. Phys. 32:878-882, 2010.

${ }^{23}$ Lakin, B. A., D. J. Grasso, S. S. Shah, R. C. Stewart, P. N. Bansal, J. D. Freedman, M. W. Grinstaff, and B. D. Snyder. Cationic agent contrast-enhanced computed tomography imaging of cartilage correlates with the compressive modulus and coefficient of friction. Osteoarthr. Cartil. 21:60-68, 2013.

${ }^{24}$ Lakin, B. A., D. J. Grasso, R. C. Stewart, J. D. Freedman, B. D. Snyder, and M. W. Grinstaff. Contrast enhanced CT attenuation correlates with the GAG content of bovine meniscus. J. Orthop. Res. 31:1765-1771, 2013.

${ }^{25}$ Lusic, H., and M. W. Grinstaff. X-ray-computed tomography contrast agents. Chem. Rev. 113:1641-1666, 2013.

${ }^{26}$ Maroudas, A., P. Bullough, S. A. Swanson, and M. A. Freeman. The permeability of articular cartilage. J. Bone Joint Surg. Br. 50:166-177, 1968.

${ }^{27}$ Mow, V. C., W. Y. Gu, and F. H. Chen. Structure and function of articular cartilage and meniscus. In: Basic Orthopaedic Biomechanics \& Mechano-biology, edited by V. C. Mow, and R. Huiskes. Philadelphia: Lippincott Williams \& Wilkins, 2005, pp. 181-258.

${ }^{28}$ Mow, V. C., M. H. Holmes, and W. M. Lai. Fluid transport and mechanical properties of articular cartilage: a review. J. Biomech. 17:377-394, 1984.

${ }^{29}$ Nieminen, H. J., T. Ylitalo, S. Karhula, J.-P. Suuronen, S. Kauppinen, R. Serimaa, E. Hæggström, K. P. H. Pritzker, M. Valkealahti, P. Lehenkari, M. Finnilä, and S. Saarakkala. Determining collagen distribution in articular cartilage using contrast-enhanced micro-computed tomography. Osteoarthr. Cartil. 23:1613-1621, 2015.

${ }^{30}$ Palmer, A. W., R. E. Guldberg, and M. E. Levenston. Analysis of cartilage matrix fixed charge density and threedimensional morphology via contrast-enhanced microcomputed tomography. Proc. Natl. Acad. Sci. USA. 103:19255-19260, 2006.

${ }^{31}$ Panula, H. E., M. M. Hyttinen, J. P. Arokoski, T. K. Långsjö, A. Pelttari, I. Kiviranta, and H. J. Helminen. Articular cartilage superficial zone collagen birefringence reduced and cartilage thickness increased before surface fibrillation in experimental osteoarthritis. Ann. Rheum. Dis. 57:237-245, 1998.

${ }^{32}$ Paschalis, E. P., F. Betts, E. DiCarlo, R. Mendelsohn, and A. L. Boskey. FTIR microspectroscopic analysis of human iliac crest biopsies from untreated osteoporotic bone. Calcif. Tissue Int. 61:487-492, 1997.

${ }^{33}$ Petersen, W., and B. Tillmann. Collagenous fibril texture of the human knee joint menisci. Anat. Embryol. (Berl) 197:317-324, 1998.

${ }^{34}$ Silvast, T. S., J. S. Jurvelin, A. S. Aula, M. J. Lammi, and J. Töyräs. Contrast agent-enhanced computed tomography of articular cartilage: association with tissue composition and properties. Acta radiol. 50:78-85, 2009. 
${ }^{35}$ Silvast, T. S., J. S. Jurvelin, M. J. Lammi, and J. Töyräs. pQCT study on diffusion and equilibrium distribution of iodinated anionic contrast agent in human articular cartilage-associations to matrix composition and integrity. Osteoarthr. Cartil. 17:26-32, 2009.

${ }^{36}$ Silvast, T. S., H. T. Kokkonen, J. S. Jurvelin, T. M. Quinn, M. T. Nieminen, and J. Töyräs. Diffusion and near-equilibrium distribution of MRI and CT contrast agents in articular cartilage. Phys. Med. Biol. 54:6823-6836, 2009.

${ }^{37}$ Stewart, R. C., P. N. Bansal, V. Entezari, H. Lusic, R. M. Nazarian, B. D. Snyder, and M. W. Grinstaff. Contrastenhanced CT with a high-affinity cationic contrast agent for imaging ex vivo bovine, intact ex vivo rabbit, and in vivo rabbit cartilage. Radiology 266:141-150, 2013.

${ }^{38}$ van Tiel, J., M. Siebelt, J. H. Waarsing, T. M. Piscaer, M. Van Straten, R. Booij, M. L. Dijkshoorn, G. J. Kleinrensink, J. A. Verhaar, G. P. Krestin, H. Weinans, and E. H. G. Oei. CT arthrography of the human knee to measure cartilage quality with low radiation dose. Osteoarthr. Cartil. 20:678-685, 2012.

${ }^{39}$ Xia, Y., J. B. Moody, H. Alhadlaq, and J. Hu. Imaging the physical and morphological properties of a multi-zone young articular cartilage at microscopic resolution. $J$. Magn. Reson. Imag. 17:365-374, 2003. 